\title{
Commentary on "Endoscopic Ultrasound (EUS)-Directed Transgastric Endoscopic Retrograde Cholangiopancreatography or EUS”
}

\author{
Se Woo Park \\ Division of Gastroenterology, Department of Internal Medicine, Hallym University Dongtan Sacred Heart Hospital, Hallym University College \\ of Medicine, Hwaseong, Korea
}

See "Endoscopic Ultrasound (EUS)-Directed Transgastric Endoscopic Retrograde Cholangiopancreatography or EUS: Mid-Term Analysis of an Emerging Procedure" by Amy Tyberg, Jose Nieto, Sanjay Salgado, et al., on page 185-190.

I read with great interest the paper by Tyberg et al. ${ }^{1}$ entitled, "Endoscopic Ultrasound (EUS)-Directed Transgastric Endoscopic Retrograde Cholangiopancreatography or EUS: MidTerm Analysis of an Emerging Procedure," published in this issue of Clinical Endoscopy. The authors reported results of what appears to be an evaluation of the efficacy and safety of the EUS-directed transgastric endoscopic retrograde cholangiopancreatography (EDGE) technique. Technical success was achieved in all 16 patients, with the gastrogastric approach in 6 and the jejunogastric approach in 10. Moreover, 10 of 11 cases achieved clinical success; the other 5 cases were awaiting fistula maturation. In terms of adverse events, stent dislodgement occurred in 3 patients and jejunal perforation during sequential endoscopic retrograde cholangiopancreatography (ERCP) procedures occurred in 1 patient. The mean weight difference before and after the procedure, the authors' greatest concern, was minimal. Finally, the authors concluded that the EDGE technique is an effective, minimally invasive, single-team, and often single-session solution to the difficulties associated with ERCP in patients who have undergone Roux-

Received: February 27, 2017 Accepted: March 3, 2017

Correspondence: Se Woo Park

Division of Gastroenterology, Department of Internal Medicine, Hallym University Dongtan Sacred Heart Hospital, Hallym University College of Medicine, 7 Keunjaebong-gil, Hwaseong 18450, Korea

Tel: +82-31-8086-2858, Fax: +82-31-8086-2029, E-mail: britnepak@hallym.or.kr

(c) This is an Open Access article distributed under the terms of the Creative Commons Attribution Non-Commercial License (http://creativecommons.org/ licenses/by-nc/3.0) which permits unrestricted non-commercial use, distribution, and reproduction in any medium, provided the original work is properly cited.
en-Y gastric bypass (RYGB).

This technique is similar to EUS-guided gastrojejunostomy, with an approach to the proximal jejunum from the stomach under endosonographic guidance, and placement of an exclusive bi-flanged lumen-apposing metal stent (LAMS) along with the previously formed fistula tract. ${ }^{2}$ In the clinical setting, the major concern with this procedure is appropriate endosonographic positioning of the proximal jejunum because it is unclear which portion of the jejunum is being scanned. ${ }^{2}$ Similarly, identification of the safest point of the bypassed portion of the stomach (the excluded stomach in this article) is critical in the EDGE technique because this may not always maintain dilation sufficient for needle puncture. In addition to target identification, the choice of appropriate devices for the creation of the anastomosis (LAMS) appears to be another critical concern throughout the EDGE procedure. In this study, stent (AXIOS ${ }^{\mathrm{m}}$; Boston Scientific, Marlborough, MA, USA) dislodgement during the index procedure requiring second revision with additional stent placement occurred in three cases. To date, two representative LAMS implants, AXIOS and SPAXUS (TaeWoong Medical, Goyang, Korea), are commercially available for various EUS-guided transmural drainage and intervention such as necrosectomy. ${ }^{3}$ The flange of the AXIOS stent for prevention of stent migration and holding the tissue layers together is generally stronger than the SPAXUS stent. The AXIOS stent also has a larger flange diameter (21 or $24 \mathrm{~mm}$ ) and lumen diameter (10 or $15 \mathrm{~mm}$ ). Nevertheless, the SPAXUS stent has a considerable advantage because it is easier to deploy than the AXIOS stent. Further- 
more, the flare (flange; 23,25 , and $31 \mathrm{~mm}$ ) and lumen (body; 8 , 10 , and $16 \mathrm{~mm}$ ) diameters are larger than those in the AXIOS. Larger luminal diameters enable the use in various indications for EUS-guided procedures. In addition, the retroflexed flanges in this stent prevent stent migration, and facilitate creation and maintenance of the stoma. ${ }^{3}$ To date, no evidence indicates which LAMS is better with regard to efficacy and safety for entero-enteric fistula formation through direct comparisons between AXIOS and SPAXUS. Thus, the choice of the appropriate LAMS for creation of the anastomosis should be made in accordance with the main purpose of fistula formation, type of sequential endoscopic procedure through the fistula, preference of the endoscopist, characteristics of the lesion, length and diameter of the stent, and type of flange for lumen-to-lumen anchoring and prevention of migration.

The authors also concluded that the EDGE technique is a single-session solution to the difficulties associated with ERCP in patients who have undergone RYGB. However, Itoi et al. demonstrated that the total duration to full stomal maturation without leakage at the anastomotic site in a pig model is approximately 1 month (27-28 days). ${ }^{3}$ This finding is similar to that of the present study, in which five patients were awaiting fistula tract maturation prior to ERCP or EUS at the time of publication. In general, metal stents partially expand immediately after deployment and then gradually (generally 2 or 3 days or more, depending on the lesion characteristics) expand to their full extent, even though the radial force may be high. ${ }^{4}$ Thus, the advantages of the EDGE technique emphasized by the authors may have limitations in terms of a single-session procedure.

Although further studies are needed to compare the efficacy and safety of direct methods such as percutaneous or EUS-guided transmural approaches with those of bridging methods such as EDGE for sequential endoscopic procedures, EDGE is a potentially valuable technique with efficacy and safety comparable with those of the current standard therapies (transoral, percutaneous, or surgical approaches). EDGE should be considered a new minimally invasive treatment modality for patients with a surgically altered anatomy.

\section{Conflicts of Interest}

The author has no financial conflicts of interest.

\section{REFERENCES}

1. Tyberg A, Nieto J, Salgado S, et al. Endoscopic ultrasound (EUS)-directed transgastric endoscopic retrograde cholangiopancreatography or EUS: mid-term analysis of an emerging procedure. Clin Endosc 2017;50:185190.

2. Tyberg A, Perez-Miranda M, Sanchez-Ocaña R, et al. Endoscopic ultrasound-guided gastrojejunostomy with a lumen-apposing metal stent: a multicenter, international experience. Endosc Int Open 2016;4:E276-E281.

3. Itoi T, Itokawa F, Uraoka T, et al. Novel EUS-guided gastrojejunostomy technique using a new double-balloon enteric tube and lumen-apposing metal stent (with videos). Gastrointest Endosc 2013;78:934-939.

4. Mangiavillano B, Pagano N, Baron TH, et al. Biliary and pancreatic stenting: devices and insertion techniques in therapeutic endoscopic retrograde cholangiopancreatography and endoscopic ultrasonography. World J Gastrointest Endosc 2016;8:143-156. 